\title{
A New Filter of Morphological Opening by Reconstruction for LiDAR Data
}

\author{
SUN Meiling, LI Yongshu, CHEN Qiang, CAI Guolin, REN Zizhen \\ Faculty of Geosciences and Environmental Engineering, Southwest Jiaotong University, Chengdu, China \\ mlsun@swjtu.edu.cn
}

\begin{abstract}
In this paper, a new filtering algorithm of LiDAR point clouds is presented, which can work well in complex cityscapes. This method generates marker image from grid DSM by erosion firstly, and then geodesic dilate on marker image repeatedly to implement opening by reconstruction process, finally white top-hat reconstruction are used to achieve the $\mathrm{nDSM}$ and classify ground and non-ground object points. When tested against the ISPRS LiDAR reference urban dataset and compared with representative filtering methods, this approach is particularly effective at minimizing Type I error and Total error rates, while maintaining acceptable Type II error rates. So this approach has good reliability and practicability in complex urban area.
\end{abstract}

Key Words - LiDAR, data filtering, morphological opening by reconstruction, white top-hat reconstruction, geodesic dilate

\section{INTRODUCTION}

The Light Detection and Ranging (LiDAR) technology can obtain high-precision 3D point clouds. Over the past few years, the generation of Digital Elevation Models (DEMs) and three-dimensional urban model from LiDAR point clouds have become the most important tasks in the field of geoinformatics. Although researchers have developed many filters to separate bare-Earth points from point clouds, the problem has not been fully solved due to the high diversity of terrain, especially on complex urban areas ${ }^{[1]}$. Therefore, developing efficient and effective methods for terrain data filtering is currently an active topic of research.

To assess the performance of various filters developed, the ISPRS Working Group III/3 conducted a test to determine the performance of these filters ${ }^{[1]}$. Of the eight algorithms originally tested, Axelsson's adaptive triangulated irregular network (TIN) model performed the best on twelve of the fifteen samples and has been implemented in the TerraScan software package ${ }^{[2]}$. The second best performing filter originally tested is the Pfeifer's hierarchical robust interpolation algorithm ${ }^{[3]}$. It performed the best on two of the fifteen samples and has been implemented in the SCOP++ software package.

Among the current filtering algorithms, interpolation-based ${ }^{[3]}$, slope-based $^{[1]}$ and morphological ${ }^{[4,5]}$ are the most popular approaches. Compared with other methods, morphological methods are conceptually simple and can be easily implemented.

The problem of using a morphological opening with larger window sizes is that it will produce a surface with more protruded terrain features flattened. Therefore, how to keep the terrain features unchanged while using large window sizes for opening is the biggest challenge.

Zhang proposed a method to remove surface objects while preserving terrain using gradually increased window sizes ${ }^{[4]}$. Chen presented a method that is similar to Zhang's method, but does not require the assumption of a constant slope ${ }^{[5]}$. Chen published the results of their algorithm run against the fifteen ISPRS samples, improving on Axelsson's algorithm for seven of fifteen samples.

The objective of this paper is to present a new morphological filter method that can remove non-ground objects and preserve terrain features during the morphological opening by reconstruction, even with large window sizes. Similar to Zhang's $\operatorname{method}^{[4]}$, progressively increased window sizes and height thresholds are used for morphological by reconstruction operations.

To evaluate its performance, this method is compared with three representative methods in the literature over urban sites with different complexity.

\section{MORPHOLOGICAL OPENING BY RECONSTRUCTION}

Mathematical morphology composes operations based on set theory to extract features from an image. Morphological opening by reconstruction is an important morphology method based on geodesic dilation. It has the effect of preserving the structures not entirely removed by opening, wiping out the others ${ }^{[6]}$.

It employs two input images that are called marker and mask images. In the following the marker image is denoted by $g$ and the mask image by $f$. Both images are identical in size, and $g \leq f$.In LiDAR data filtering of morphological opening by reconstruction, the mask image is grid DSM and the marker image is mask image by erosion with structure element $(b)$ :

$$
g=\varepsilon_{b}(f)=f \Theta b
$$

The symbol $\Theta$ is used for the erosion operation. The classical morphology dilation of $g$ with structuring element $\mathrm{b}$ is given by

$$
\delta(g)=g \oplus b
$$

The symbol $\oplus$ is used for the dilation operation. The geodesic dilation of size 1 of the marker image $g$ with respect to mask image $\mathrm{f}$ is defined as:

$$
\delta_{f}^{(1)}(g)=(g \oplus b) \wedge f
$$

In this equation, $\wedge$ stands for the point-wise minimum between the dilated marker image and the mask image, $g \oplus b$ is the dilation of $g$ with the elementary isotropic structuring element $b$. The geodesic dilation of size $\mathrm{n}$ of the marker image $\mathrm{g}$ 
with respect to a mask image $f$ is obtained by performing $n$ successive geodesic dilation of size 1 of $g$ with respect to $f$ :

$$
\delta_{f}^{(n)}(g)=\underbrace{\delta_{f}^{(1)}(g) \circ \delta_{f}^{(1)}(g) \circ \cdots \circ \delta_{f}^{(1)}(g)}_{n \text { times }}
$$

Equation 4 defines the morphological opening by reconstruction with geodesic dilation of the mask $f$ from marker the $g$. Therefore, the morphological opening by reconstruction based on geodesic dilation is defined as:

$$
\gamma_{R}^{(b)}(f)=\delta_{f}^{(i)}\left(g \neq \oint^{\prime}\right)[\varepsilon f
$$

In this equation, $i$ is the repeated times while $\delta_{f}^{(i)}(g)=\delta_{f}^{(i+1)}(g)$.

The $n D S M$ can be got with white top-hat reconstruction. Based on opening by reconstruction, it can be defined as:

$$
n D S M=f-\gamma_{R}^{(b)}(f)
$$

Morphological opening by reconstruction of geodesic dilations of a 1D signal is illustrated as Figure 1. They are mask image, $\mathrm{nDSM}$ with erosion radius $w=8, w=30$ and constant threshold $e t=0.5 \mathrm{~m}$.

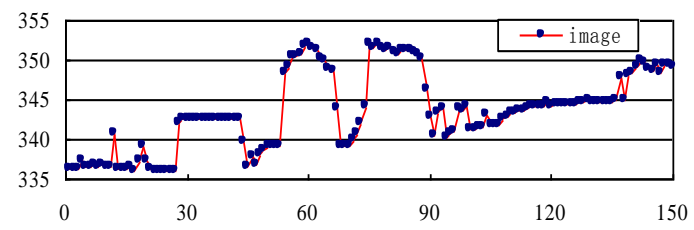

(a) mask

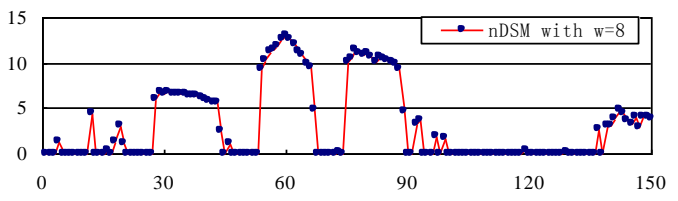

(b) nDSM with $\mathrm{w}=8$

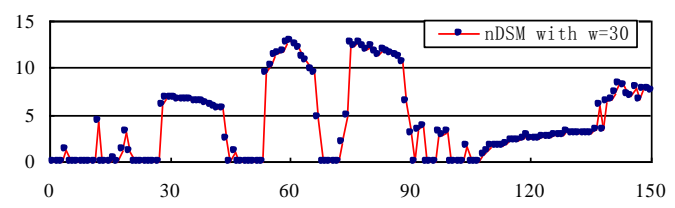

(c) $\mathrm{nDSM}$ with $\mathrm{w}=30$

Fig.1 Different nDSMs based on morphological opening by reconstruction with different erosion structure element

From Figure 1, we can see that with constant height threshold (et) value, if structure element is small, the objects will not be filtered completely. If structure element is large, the objects will be filtered with slope ground considered as non-ground points. The selection of a filtering window size is a critical factor for this method. An ideal window size does not exist in the real world. To settle the problem and minimize Type I errors, progressively increased window sizes and height thresholds used by the progressive filter of morphological opening by reconstruction is similar to Zhang's method ${ }^{[4]}$.

\section{PROGRESSIVE MORPHOLOGICAL OPENING BY RECONSTRUCTION}

The progressive filter framework of morphological opening by reconstruction based on geodesic dilation is outlined in Figure 2.

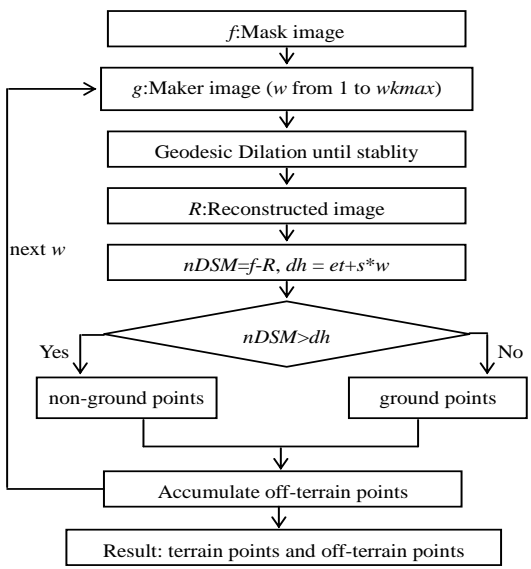

Fig.2 Progressive filter framework of morphological opening by reconstruction

From Figure 2, we can see that the input to this progressive filter process is the mask image. In the first step, a marker image is generated with respect to the mask image. The marker image is generated by erosion with disk structure element from the mask image. To avoid problems caused by an improperly selected element size, we propose to use a sequence of element radius size to create a sequence of marker images.

The next step is to calculate the geodesic dilation of size 1 of the marker image with respect to the mask image. This process will be continued until the pixel values do not change any more by a further geodesic dilation of the marker image. The result of the successively performed geodesic dilations is the morphologically reconstructed image. By subtracting the reconstructed image from the mask image, the normalized DSM $(\mathrm{nDSM})$ is obtained. The classification of ground and non-ground points is carried out by the binarization of the nDSM. In the nDSM, any point above threshold value $(d h)$ is collected as a non-ground point. The $d h$ is decided by initial height threshold $(e t)$ value, erosion radius size $(w)$ and terrain slope $(s)$ value.

These steps from creating the marker image to the classification of the non-ground points will be repeated for all marker images produced with different disk erosion structure element of radius size $(w)$ values. The classification takes the results of previous iterations into account by merging it with the classified non-ground points of the current iteration. At the end of the process, the classification result represents ground and non-ground points. 


\section{IV.THE PROPOSED SCHEME}

The detailed steps to use the progressive filter of morphological opening by reconstruction are shown in Figure 3 and given as follows.

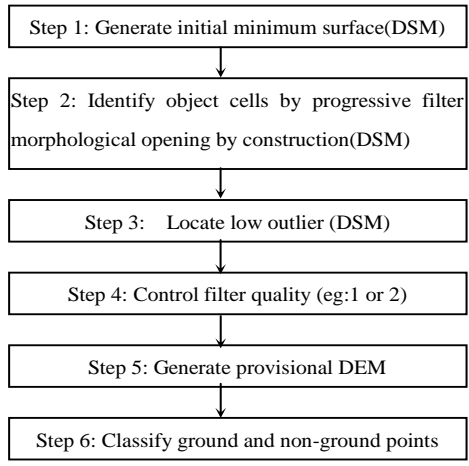

Fig.3 Workflow diagram of the progressive filter method

Step 1: The point clouds of irregularly spaced LiDAR measurements are loaded. A regularly spaced minimum surface grid is constructed by selecting the minimum elevation in each grid cell. If a cell contains no measurements, it is assigned the value by spring-metaphor inpainting technique ${ }^{[7]}$.

Step 2: It is common in LiDAR point clouds to have a small number of outliers which may be either above or below the terrain surface. While above-ground outliers could be filtered during the normal algorithm routine, the below-ground outliers require a separate approach. The morphological opening by reconstruction filter method is used to locate low outlier with inverting the point cloud in the $\mathrm{z}$-axis and applying the filter with parameters $(w=1, d h=5 \mathrm{~m})$.

Step 3: The progressive filter of morphological opening by reconstruction whose major component are erosion and geodesic dilation operation is applied to the grid surface. In the iterations, the minimum elevation surface and a gradually increased window size are used as input for the filter. The output of this step includes the detected non-ground points based on the $d h$.

Step 4: To control filter quality, we utilize morphological opening with small structure element $(w=1$ or 2$)$ after progressive filter of morphological opening by reconstruction. The output of this step includes the detected non-ground points based on the initial height elevation threshold $(e t)$.

Step 5: The end result of the process described above is a binary grid where each cell is classified as being either bare earth (BE) or object $(\mathrm{OBJ})$. The algorithm then applies this mask to the starting minimum surface to eliminate non-ground cells. These cells are then inpainted according to the same process described previously, producing a provisional DEM.

Step 6: The final step of the algorithm is the identification of BE/OBJ LiDAR points. This is accomplished by measuring the vertical distance between each LiDAR point and the provisional DEM, and applying a threshold (et) calculation.

\section{V.EXPERIMENT AND RESULTS}

For this research, nine datasets over urban areas, as well as their respective reference (ground-truth) data, were acquired from the ISPRS Commission III/WG $3^{[8]}$. These datasets are located four study sites over the Stuttgart city center with point spacing of 1 to $1.5 \mathrm{~m}$. These areas were chosen because of their diverse feature content (buildings, vegetation, railroads, open fields, bridges, etc.) and the filtering difficulties (outliers, object complexity, attached objects, etc.).

We follow the quantitative assessment in ISPRS filter test to validate the proposed scheme ${ }^{[8]}$. The Type I, Type II, and Total errors were calculated for each sample. The Type I error is the percentage of bare earth returns misclassified as object returns. The Type II error is the percentage of object returns misclassified as bare earth returns. The Total error rate is equal to the sum of all mistaken classifications divided by the total number of points in the dataset.

The results for Total errors are compared with three representative methods in the literature ${ }^{[2,3,5]}$. The results are shown in Table 1 with uniform initial height threshold et $=0.5 \mathrm{~m}$, respective terrain slope $s=0.2 、 0.0 、 0.1 、 0.1 、 0.1 、 0.1 、 0.0$ 、 $0.1 、 0.0$, respective maximum window disk radius $w k m a x=20$ 、 20、20、20、15、10、15、20、50.

Tab.1 Comparison of Total Errors for ISPRS nine urban areas

\begin{tabular}{ccccc}
\hline Sample & $\begin{array}{c}\text { Axelsson } \\
(\boldsymbol{\%})\end{array}$ & $\begin{array}{c}\text { Pfeifer } \\
(\boldsymbol{\%})\end{array}$ & $\begin{array}{c}\text { Chen } \\
(\boldsymbol{\%})\end{array}$ & $\begin{array}{c}\text { We } \\
(\boldsymbol{\%})\end{array}$ \\
\hline Samp11 & $\underline{\mathbf{1 0 . 7 6}}$ & 17.35 & 13.92 & 13.85 \\
Samp12 & 3.25 & 4.50 & 3.61 & $\underline{\mathbf{2 . 9 3}}$ \\
Samp21 & 4.25 & 2.57 & 2.28 & $\underline{\mathbf{1 . 0 6}}$ \\
Samp22 & 3.63 & 6.71 & $\underline{\mathbf{3 . 6 1}}$ & 4.57 \\
Samp23 & $\underline{\mathbf{4 . 0 0}}$ & 8.22 & 9.05 & 4.36 \\
Samp24 & 4.42 & 8.64 & $\underline{\mathbf{3 . 6 1}}$ & 4.32 \\
Samp31 & 4.78 & 1.80 & $\underline{\mathbf{1 . 2 7}}$ & 1.33 \\
Samp41 & 13.91 & 10.75 & 34.03 & $\underline{\mathbf{5 . 4 2}}$ \\
Samp42 & 1.62 & 2.64 & 2.20 & $\underline{\mathbf{0 . 9 7}}$ \\
Mean of total & 5.62 & 7.02 & 8.18 & $\underline{\mathbf{4 . 3 1}}$ \\
Mean of type I & 7.72 & 10.74 & - & $\underline{\mathbf{3 . 4 3}}$ \\
Mean of type II & 4.94 & $\underline{\mathbf{2 . 8 1}}$ & - & 5.88 \\
\hline No: The
\end{tabular}

Note: The algorithms with the lowest total error are underlined.

The comparison indicates our method obtained the lowest total errors for four samples and the remaining five samples are close to the lowest errors. It improved Axelsson's algorithm performance on six samples. The mean of Type I and Total error are minimum compared to traditional filtering methods with Type II error increased not remarkably. Due to of the variety of the ISPRS test data, the results indicate that the proposed method is robust to different city landscape types.

Visual inspection results show that the object and bare ground are not only classified well, but also it keeps details of terrain. For instance, Figure 4 shows the processing result for sample 12 and 22, which contains buildings, trees, cars and a 
crossover bridge in an urban site. At the same time, some objects are not separated correctly. It accords with ISPRS suggestion that filtering should be biased in favor of minimizing Type I errors, because Type II errors will be easier to correct manually through visual inspection. So it makes clear that our filter has good feasibility and reliability.

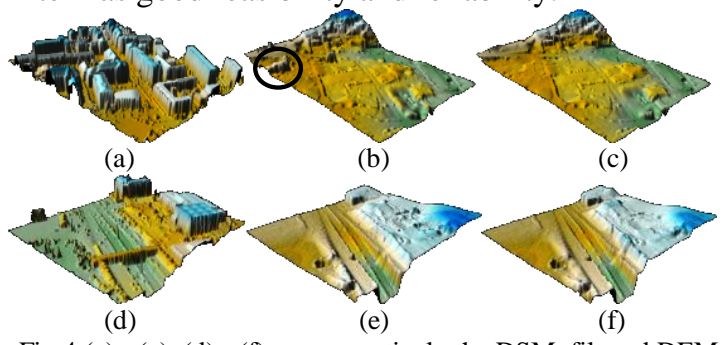

Fig.4 (a) - (c), (d) - (f) are respectively the DSM, filtered DEM, and true DEM for sample 12 and 22

\section{CONCLUSION}

In this paper, a new morphological filter was built to separate non-ground points from ground points in LiDAR data. The basis of the filter is a morphological opening by reconstruction process which employs geodesic dilation.

The experimental results for ISPRS data show that this approach is able to classify ground and non-ground points effectively in complex cityscapes. The mean of Type I and Total error are minimum compared to traditional filtering methods with Type II error increased not obviously. Future work may also focus on the non-ground point classification process.

\section{ACKNOWLEDGEMENTS}

This work was supported by the Natural Science Foundation of China (Grant Nos. 51178404, 41072220), Specialized Research Fund for the Doctoral Program of Higher Education (Grant No. 20100184110019), Fundamental Research Funds for the Central Universities (Grant Nos.SWJTU09BR050, 2682013CX009)

\section{REFERENCES}

[1] Sithole G, Vosselman G. Experimental comparison of filter algorithms for bare-Earth extraction from airborne laser scanning point clouds[J]. ISPRS Journal of Photogrammetry and Remote Sensing. 2004, 59(1-2):85-101.

[2] Axelsson P. DEM generation from laser scanner data using adaptive TIN Models[J].International Archives of Photogrammetry and Remote Sensing.2000, 33(B4/1):110-117.

[3] Pfeifer, N., Stadler, P., Briese, C. Derivation of digital terrain models in the SCOP environment. Proc. OEEPE workshop on Airborne Laser scanning and Interferometric SAR for Detailed Digital Elevation Models, March, 2001,OEEPE Publication no. 40, 13 pp. (on CD-ROM).

[4] Zhang K, Chen S C, Whitman D, et al. A progressive morphological filter for removing nonground measurement from LiDAR Data [J]. IEEE Transactions on Geoscience and Remote
Sensing, 2003, 41(4): 872-882.

[5] Chen Q, Gong P, Dennis B, and Xie G. Filtering airborne laser scanning data with morphological methods[J]. Photogrammetric Engineering and Remote Sensing, 2007, 73(2):175-185.

[6] Soille P. Morphological image analysis, principles and applications[M]. Berlin:Springer-Verlag, 2003.

[7] D’Errico J. Inpaint_nans.m. http://www.mathworks.com/matlabcentral/fileexchange/4551, 2004.

[8] Sithole G, Vosselman G. The full Report: ISPRS comparison of filters[R]. http://www. itc.nl /isprswgIII-3/filtertest/, 2003. 